\title{
DECOMPOSITION OF OPENNESS
}

\author{
C.W. BAKER \\ Department of Mathematics \\ Indiana University Southeast \\ New Albany, Indiana 47150
}

(Received October 23, 1992)

ABSTRACT. In this note weak openness and almost openness are used to develop two decompositions of openness. Two additional conditions related to openness also are developed.

KEY WORDS AND PHRASES. Decomposition, openness, weak openness, almost openness.

1980 AMS SUBJECT CLASSIFICATION CODE. 54C10

\section{PRELIMINARIES}

The symbols $\mathrm{X}$ and $\mathrm{Y}$ denote topological spaces with no separation axioms assumed unless explicitly stated. The interior and closure of a set $U$ are denoted by Int $U$ and $\mathrm{Cl} U$, respectively.

DEFINITION 1. [1]. A function $f: X-Y$ is said to be weakly open if $f(U) \subseteq$ Int $f(C l U)$ for every open subset $U$ of $X$.

DEFINITION 2. [1]. A function $f: X=Y$ is said to be almost open if $f(U) \subseteq$ Int $C l f(U)$ for every open subset $U$ of $X$.

2. RELATIVELY OPEN CONDITIONS

In this section the concept of relative openness is used to develop two near open conditions. These conditions are then used to to obtain decompositions of openness. The method is similar to that used by Chew and Tong in [2] to obtain a decomposition of continuity. DEFINITION 3. A function $\mathrm{f}: \mathrm{X} \rightarrow \mathrm{Y}$ is said to be relatively weakly open provided that $f(U)$ is open in $f(C l U)$ for every open subset $U$ of $X$.

Obviously openness implies relative weak openness. We shall see in Example 1 that the converse implication does not hold.

THEOREM 1. A function $\mathrm{F}: \mathrm{X} \rightarrow \mathrm{Y}$ is open if and only if $\mathrm{f}$ is weakly open and relatively weakly open.

PROOF. Assume $f$ is weakly open and relatively weakly open. Let $U$ be an open subset of $X$ and let $y \equiv F(U)$. Since $f$ is relatively weakly open, there is an open subset $V$ of $Y$ for which $f(U)=$ 
$\mathrm{E}(\mathrm{Cl} U)$ il $\mathrm{V}$. Because $\mathrm{f}$ is weakly open, it follows that $\mathrm{f}(\mathrm{U}) \subseteq$ Int $\mathrm{f}(\mathrm{Cl} U)$. Then $\mathrm{Y} \equiv \mathrm{V} \sqcap$ Int $\mathrm{f}(\mathrm{Cl} U) \equiv \mathrm{V} \sqcap \mathrm{f}(\mathrm{Cl} U)=\mathrm{f}(\mathrm{U})$ and therefore $f(U)$ is open.

The proof of the converse is immediate.

The following examples show that weak openness and relative weak openness are independent.

EXAMPLE 1. Let $\vec{I}$ be the discrete topology on the positive real numbers, $\mathbb{F}^{+}$, and it, the usual topology on the real numbers, $F_{\text {. }}$ Define $\mathrm{f}:\left(\mathbb{F}^{+}, \bar{I}_{1}\right)-(\mathbb{F},+j)$ by

$$
f(x)=\left[\begin{array}{r}
x \text { if } x \text { is rational } \\
-x \text { if } x \text { is irrational }
\end{array}\right.
$$

Since $\mathrm{E}(\mathrm{U})=\mathrm{E}(\mathrm{Cl} \mathrm{U})$ for any subset $U$ of $\mathbb{F}^{+}$, $\mathrm{f}$ is relatively weakly open. However, $\mathrm{f}$ is not weakly open because Int $\mathrm{f}(\mathrm{Cl} U)=$ for any subset $U$ of $\mathbb{F}^{+}$.

EXAMPLE 2. [1]. Let $X=\{a, b, c\}, \Xi=\{X, s,\{a\},\{c\},\{a, c\}\}$ and $: t=\{X, a,\{b\},\{a, b\},\{b, c\}\}$. Let $f:(X, \xi) \rightarrow(X, g)$ be the identity function. From [1] $f$ is weakly open. To see that $f$ is not relatively weakly open, observe that for $U=\{a\}, f(C l U)=\{a, b\}$ and $\mathrm{E}(\mathrm{U})$ is not open in $\mathrm{E}(\mathrm{Cl} U)$.

DEFINITION 4. A function $f: X-Y$ is said to be relatively almost open if $\mathrm{E}(U)$ is open in $\mathrm{Cl} E(U)$ for every open subset $U$ of $X$. Clearly every open function is relatively almost open. However, we shall see that the function in Example 2 is relatively almost open but not open.

THEOREM 2. A function $f: X \div Y$ is open if and only if $f$ is almost open and relatively almost open.

PROOF. Assume $\mathrm{f}$ is almost open and relatively almost open. Let $U$ be an open subset of $X$ and let $Y \equiv f(U)$. Since $f$ is relatively almost open, there exists an open subset $V$ of $Y$ for which $f(U)=$ $V \cap \mathrm{Cl} f(U)$. Because $f$ is almost open, we have that $f(U) \subseteq$

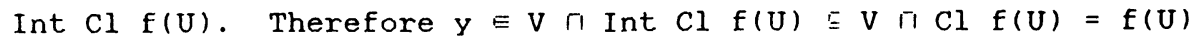
and hence $f(U)$ is open.

The converse is obvious.

The function is Example 2 is relatively almost open, since $f(U)$ $=C l E(U)$ for every open subset $U$ of $X$, but is not almost open, because Int $\mathrm{Cl} f(U)=$. for every open subset $U$ of $X$. Therefore relative almost openness does not imply almost openness. The following example shows that the converse implication also fails.

EXAMPLE 3. Assume $\mathbb{F}^{+}$and $\mathbb{F}$ have their usual topologies. Define the function $\mathrm{E}: \mathbb{F}^{+} \Rightarrow \mathbb{F}^{\text {by }}$

$$
f(x)=\left\{\begin{array}{r}
x \text { if } x \text { is rational } \\
-x \text { if } x \text { is irrational }
\end{array}\right.
$$

If $U=(0,1)$, then $C l f(U)=[1,-1]$ and $f(U)$ is not open in $C l \mathrm{f}(\mathrm{U})$. Therefore $\mathrm{E}$ is not relatively almost open. However, it is clear that for any open interval (and hence for any open set) $U$ that $\mathrm{f}(\mathrm{U}) \subseteq$ Int $\mathrm{Cl} \mathrm{f}(\mathrm{U})$. Hence $\mathrm{f}$ is almost open. 
3. INTERIORITY CONDITIONS

In this section we define two additional near open conditions. These conditions when combined with weak openness in one case and almost openness in the other imply openness. The conditions are analogous to the interiority condition defined by chew and Tong in [2].

DEFINITION 5. A function $F: X-Y$ is said to satisfy the weakly open interiority condition if Int $f(C l U) \subseteq f(U)$ for every open subset $U$ of $X$.

THEOREM 3. If $\mathrm{E}: \mathrm{X}$; $\mathrm{Y}$ is weakly open and satisfies the weakly open interiority condition, then $f$ is open.

PROOF. Let $U$ be an open subset of $X$. Since $f$ is weakly open $\mathrm{f}(\mathrm{U}) \cong$ Int $\mathrm{f}(\mathrm{Cl} U)$. However, because $f$ satisfies the weakly open interiority condition, $f(U)=$ Int $f(C l U)$ and therefore $f(U)$ is open. DEFINITION 6. A function $\mathrm{F}$ : $\mathrm{X}-\mathrm{Y}$ is said to satisfy the almost open interiority condition provided that Int $\mathrm{Cl} f(U) \subseteq f(U)$ for every open subset $U$ of $X$.

THEOREM 4. If $\mathrm{f}: \mathrm{X} \rightarrow \mathrm{Y}$ is almost open and satisfies the almost open interiority condition, then $f$ is open.

The proof is analogous to that of Theorem 3 and is omitted.

The following example shows that neither of these interiority conditions yields a decomposition of openness.

EXAMPLE 4. Let $X=\{a, b, c\}, \cong=\{x, a,\{a\}\}$ and $i^{-}=$ $\{x, a,\{a\},\{a, b\}\}$. Let $f:(X, \Xi)-(x, j)$ be the identity mapping and let $U=\{a\}$. Since Int $f(C l U)=$ Int $f(X)=\operatorname{Int} X=X \pm f(U)$, $f$ does not satisfy the weakly open interiority condition. Also, since Int $\mathrm{Cl} f(U)=$ Int $\mathrm{Cl} X=X \pm \mathrm{f}(\mathrm{U})$, $\mathrm{f}$ does not satisfy the almost open interiority condition. However, $f$ is clearly open.

\section{REFERENCES}

1. Rose, D.A. Weak openness and almost openness, Internat. J. Math. Math. Sci. $7(1984), 35-40$.

2. Chew, J. and Tong, J. Some remarks on weak continuity, Amer. Math. Monthly 98 (1991), 931-934. 


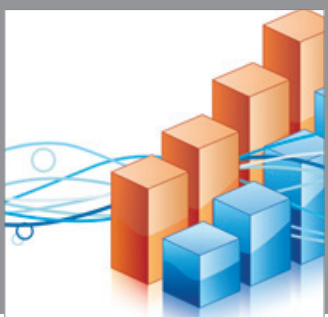

Advances in

Operations Research

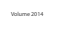

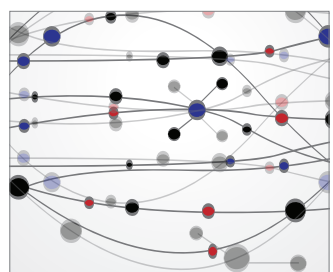

\section{The Scientific} World Journal
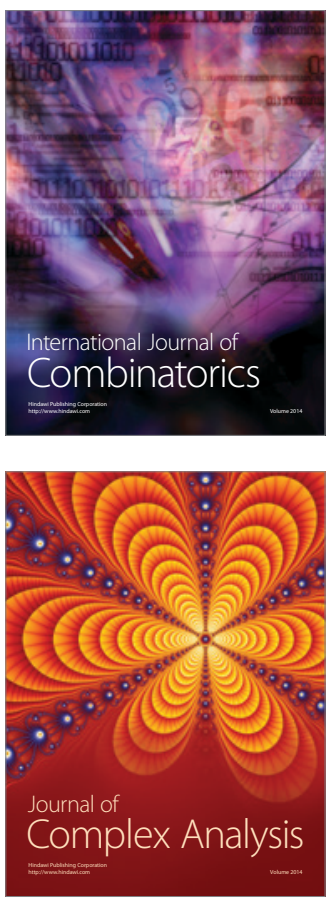

International Journal of

Mathematics and

Mathematical

Sciences
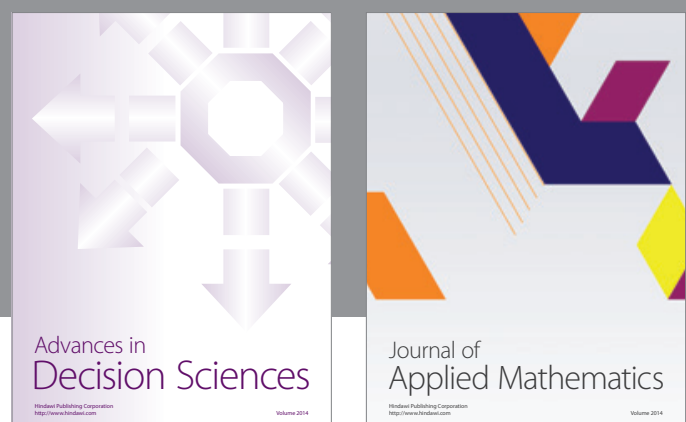

Journal of

Applied Mathematics
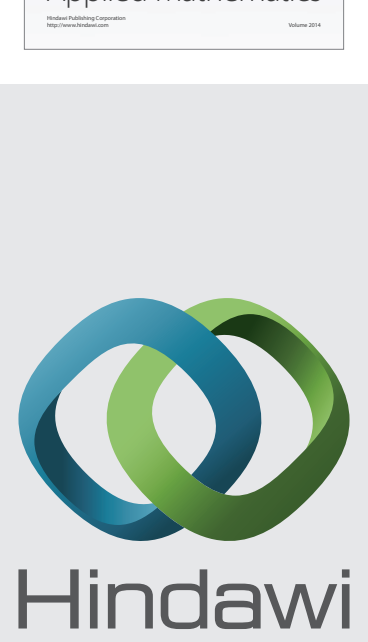

Submit your manuscripts at http://www.hindawi.com
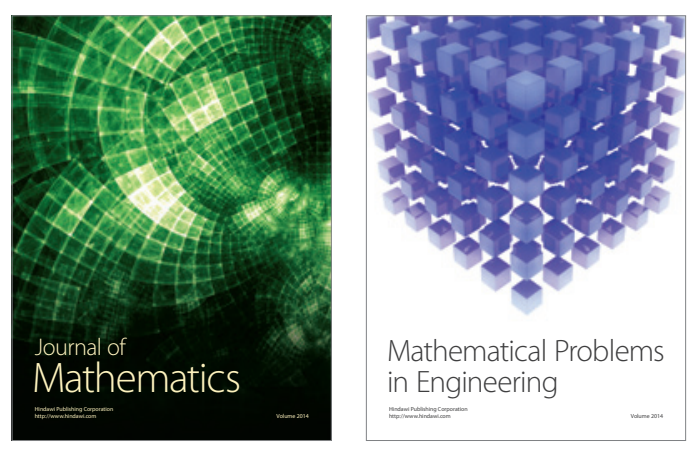

Mathematical Problems in Engineering
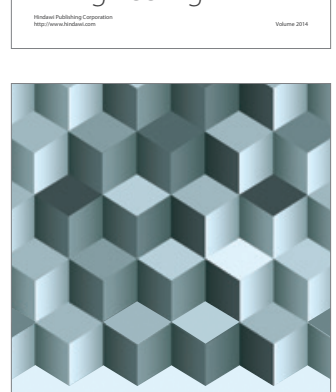

Journal of

Function Spaces
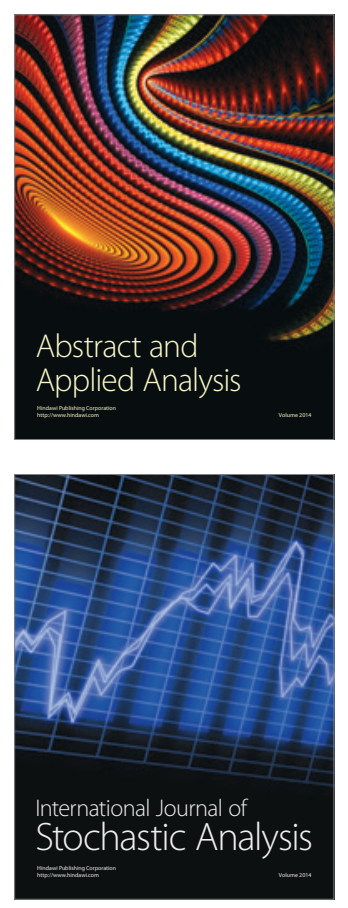

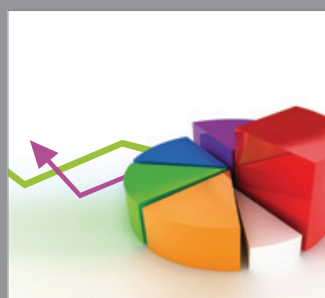

ournal of

Probability and Statistics

Promensencen
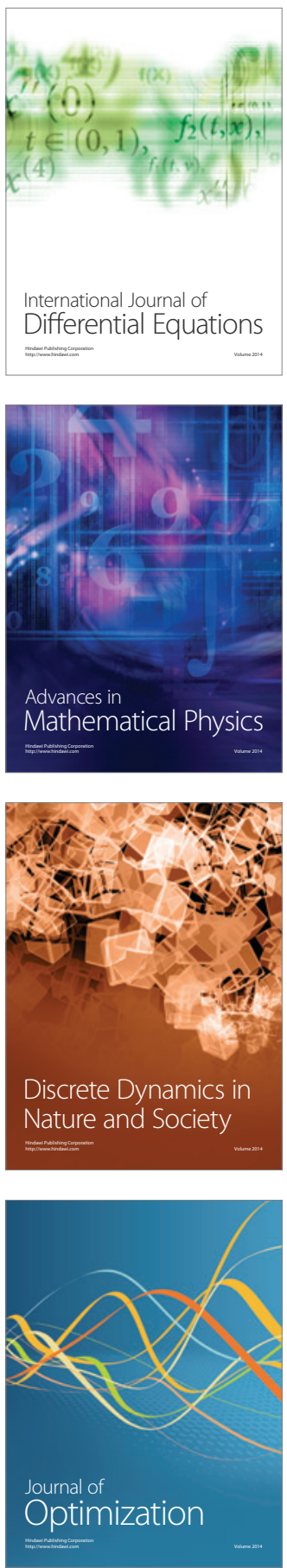